\title{
Neutrino and antineutrino CCQE scattering in the SuperScaling Approximation from MiniBooNE to NOMAD energies
}

\author{
G.D. Megias ${ }^{a}$, J.E. Amaro ${ }^{b}$, M.B. Barbaro ${ }^{\text {c,* }}$, J.A. Caballero ${ }^{\text {a }}$, T.W. Donnelly ${ }^{\mathrm{d}}$ \\ a Departamento de Física Atómica, Molecular y Nuclear, Universidad de Sevilla, 41080 Sevilla, Spain \\ b Departamento de Física Atómica, Molecular y Nuclear and Instituto Carlos I de Física Teorica y Computacional, Universidad de Granada, 18071 Granada, Spain \\ c Dipartimento di Fisica, Università di Torino and INFN, Sezione di Torino, Via P. Giuria 1, 10125 Torino, Italy \\ d Center for Theoretical Physics, Laboratory for Nuclear Science and Department of Physics, Massachusetts Institute of Technology, Cambridge, MA 02139, USA
}

\section{A R T I C L E I N F O}

\section{Article history:}

Received 30 May 2013

Received in revised form 28 June 2013

Accepted 1 July 2013

Available online 5 July 2013

Editor: W. Haxton

\begin{abstract}
A B S T R A C T
We compare the predictions of the SuperScaling model for charged-current quasielastic muonic neutrino and antineutrino scattering from ${ }^{12} \mathrm{C}$ with experimental data spanning an energy range up to $100 \mathrm{GeV}$. We discuss the sensitivity of the results to different parametrizations of the nucleon vector and axialvector form factors. Finally, we show the differences between electron and muon (anti)neutrino cross sections relevant for the $\nu$ STORM facility.
\end{abstract}

(c) 2013 Elsevier B.V. All rights reserved.

\section{Introduction}

Charged-current (CC) quasielastic (QE) muonic neutrino and antineutrino cross sections on a ${ }^{12} \mathrm{C}$ target have been recently measured by the MiniBooNE Collaboration at Fermilab [1,2] for neutrino energies in the $1 \mathrm{GeV}$ region, the neutrino and antineutrino fluxes peaking at $0.79 \mathrm{GeV}$ and $0.66 \mathrm{GeV}$, respectively, and going from 0 to about $7 \mathrm{GeV}$ with the most important contributions coming from below $3 \mathrm{GeV}$. The results have stimulated many theoretical studies [3-15] that attempt to explain the discrepancy between the data and traditional nuclear models; these include the Relativistic Fermi Gas (RFG) model, RPA calculations, the use of realistic spectral functions, relativistic Green's function approaches and relativistic mean field theory.

An empirical solution to this puzzle, proposed by the MiniBooNE Collaboration, advocates a value of the nucleon axialvector dipole mass $M_{A} \simeq 1.35 \mathrm{GeV} / c^{2}$ [1], which is significantly larger than the standard value $M_{A}=1.032 \mathrm{GeV} / c^{2}$. On the other hand microscopic explanations based on multi-nucleon excitations, in particular two-particle emission, were proposed in $[3,4,7,13]$. Those of [7,13], although rather different in their basic ingredients, have been shown to give very good agreement with the MiniBooNE data, while those of $[3,4]$, which are based on the exact relativistic evaluation of the Meson-Exchange Currents (MEC) within the 2p2h RFG approach, provide an enhancement of the cross sections but do not fully account for the discrepancy. It should be stressed

\footnotetext{
* Corresponding author.

E-mail address: barbaro@to.infn.it (M.B. Barbaro).
}

that a consistent evaluation of the MEC contribution is technically hard to achieve and an exact relativistic gauge invariant calculation of both vector and axial-vector contributions to MEC in neutrino scattering is not yet available.

On the other hand, CCQE $v_{\mu}$ and $\bar{v}_{\mu-}{ }^{12} \mathrm{C}$ cross section measurements from the NOMAD Collaboration [16] for higher beam energies, going from 3 to $100 \mathrm{GeV}$, do not call for an anomalously large axial-vector mass and do not appear to match with the lower-energy MiniBooNE results, as shown in Fig. 1 where the two sets of data are displayed. It should also be mentioned that recent data on CCQE $v_{\mu}$ - and $\bar{v}_{\mu^{-}}{ }^{12} \mathrm{C}$ from the MINERvA Collaboration $[17,18]$ are claimed to disfavor the value $M_{A} \simeq 1.35 \mathrm{GeV} / c^{2}$. It is thus desirable to perform a consistent theoretical analysis of the cross sections in the entire $0-100 \mathrm{GeV}$ energy range, using a nuclear model which can be applied up to very high energies. Such a model must obviously fulfill two basic requirements: it has to be relativistic and it must successfully describe QE electron scattering data from intermediate up to very high energies.

The SuperScaling (SuSA) model, based on the superscaling function extracted from $\mathrm{QE}$ electron scattering data, does a reasonable job of satisfying both of the above requirements: it is fully relativistic and has been constructed using those data as input. On the one hand, its applicability may be questioned at very low energies (meaning by that, momentum transfers $q \leqslant 400 \mathrm{MeV} / c$ and energy transfers $\omega \leqslant 50 \mathrm{MeV}$ ), where collective effects which violate scaling dominate. In discussing the results found (see below) we shall return to comment on this issue. On the other hand the SuSA approach can be safely extended up to very high energies, since it is based on $\left(e, e^{\prime}\right)$ data in a range going from intermediate to high energies and momentum transfers [19-22]. 


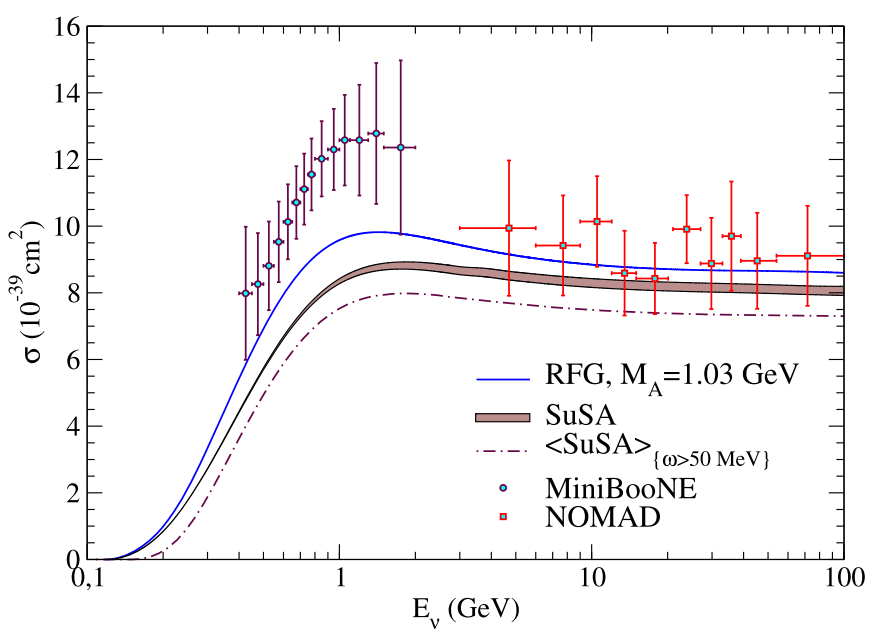

Fig. 1. (Color online.) CCQE $v_{\mu}-{ }^{12} \mathrm{C}$ cross section per nucleon displayed versus neutrino energy $E_{v}$ and evaluated using the SuSA model (brown band) with the standard value of the axial-vector dipole mass $M_{A}=1.032 \mathrm{GeV} / c^{2}$. Results are also shown for the RFG model with $M_{A}=1.032 \mathrm{GeV} / c^{2}$ (blue solid curve) and compared with the MiniBooNE [1] and NOMAD [16] experimental data. Also presented for reference are the results for SuSA excluding all contributions coming from transferred energies below $50 \mathrm{MeV}$ (dot-dashed curve).

In summary, the model gives a very good representation of all existing QE electron scattering data for high enough momentum and energy transfers, to the extent that quasielastic scattering can be isolated. Additionally, the same scaling approach has been shown to be very successful when extended to higher energies into the non-QE regime where inelastic contributions dominate [23]. However, it does not account for the typically $10-20 \%$ scaling violations that occur mainly in the transverse channel and are associated with non-impulsive processes induced by two-body MesonExchange Currents (MEC) (see [24-27]). These should therefore be added to obtain a representation of all of the contributions to the inclusive cross section in the relevant kinematical regions. While these have been included in our past studies of the differential neutrino cross sections, where one can be sure that the momentum and energy transfers are sufficiently large that the modeling is robust, in this Letter we neglect such contributions since, as discussed below, the low- $q /$ low- $\omega$ region is important for the total cross section. We are not confident of the validity of the present $2 \mathrm{p} 2 \mathrm{~h}$ MEC model in this kinematic region - work is in progress to correct this deficiency.

The SuSA model has been extensively described in previous work (see, e.g., [28]). In this Letter we only summarize the basic ideas and focus on applying them to CCQE (anti)neutrino scattering from ${ }^{12} \mathrm{C}$, comparing the results with the MiniBooNE and NOMAD data. We also study the sensitivity of the cross section to different up-to-date parametrizations of the nucleon form factors entering the cross section, $G_{E}, G_{M}$ and $G_{A}$, studying in particular the effects of a monopole parametrization for the axial-vector form factor. Finally, we present the SuSA predictions for electron neutrino and antineutrino cross sections, with particular reference to the vSTORM kinematical conditions [29].

\section{Results}

The SuSA approach to neutrino scattering is based on the idea of using electron scattering data to predict CC neutrino cross sections. The model was proposed in [28], where it is presented in detail. In summary, a phenomenological superscaling function, extracted from $\mathrm{QE}\left(e, e^{\prime}\right)$ data within a fully relativistic framework and embodying the essential nuclear dynamics, is multiplied by

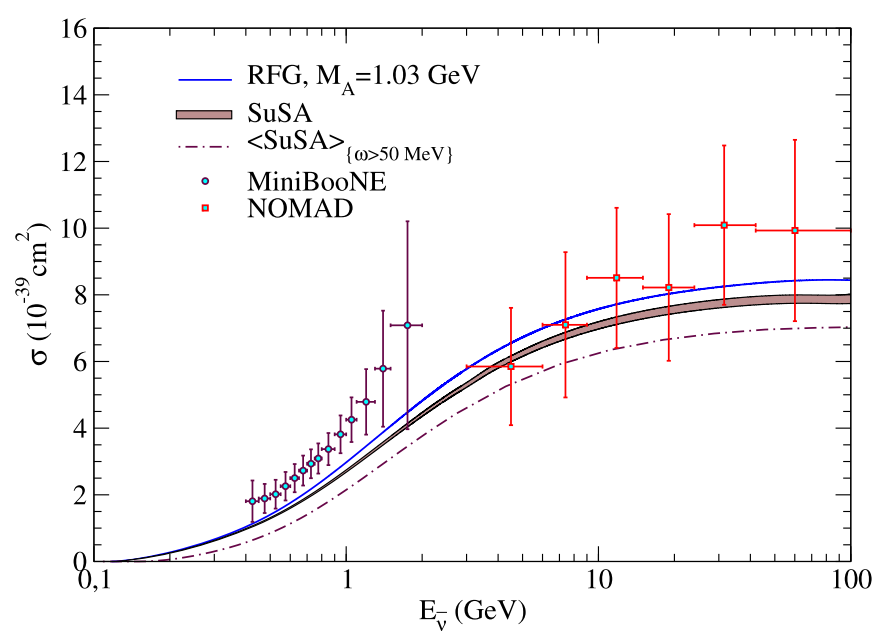

Fig. 2. (Color online.) As for Fig. 1, but now for $\bar{v}_{\mu}-{ }^{12} \mathrm{C}$ scattering. The MiniBooNE data are from [2].

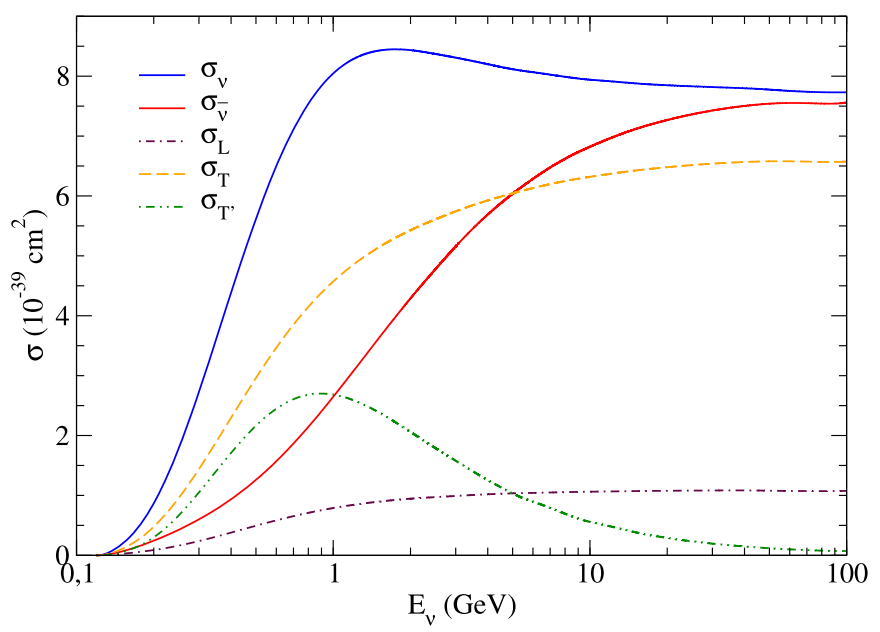

Fig. 3. (Color online.) Separated contributions in the SuSA model.

the appropriate charge-changing $N \rightarrow N$ weak interaction cross sections in order to obtain the various response functions contributing to the inclusive $\left(v_{l}, l\right)$ cross section, $R_{L}, R_{T}$ and $R_{T^{\prime}}$, each response being a combination of vector and axial-vector components.

In Figs. 1 and 2 we compare the MiniBooNE and NOMAD QE data on ${ }^{12} \mathrm{C}$ for $v_{\mu}$ and $\bar{v}_{\mu}$ scattering, respectively, with the results of the RFG and SuSA models using the standard value $M_{A}=$ $1.032 \mathrm{GeV} / c^{2}$ for the nucleon axial-vector dipole mass. The SuSA results are represented by a narrow band, corresponding to the uncertainty linked to the use of two fits of the phenomenological scaling function $[20-22,28]$. We observe that if the standard axialvector mass is used the two models underestimate the MiniBooNE data for both neutrino and antineutrino scattering while they are both quite compatible with the NOMAD data.

In Fig. 3 we show the breakdown of the neutrino and antineutrino cross sections into individual $L, T$ and $T^{\prime}$ contributions, with the last occurring as a positive (constructive) term in the neutrino cross section and a negative (destructive) term in the antineutrino cross section. Upon examining the results displayed in Figs. 1 and 2 we note that were this $V A$ interference is a bit larger, for instance via inclusion of contributions that go beyond the impulse approximation (see above), then better agreement with the neutrino data in the region of the MiniBooNE kinematics could be obtained, since 


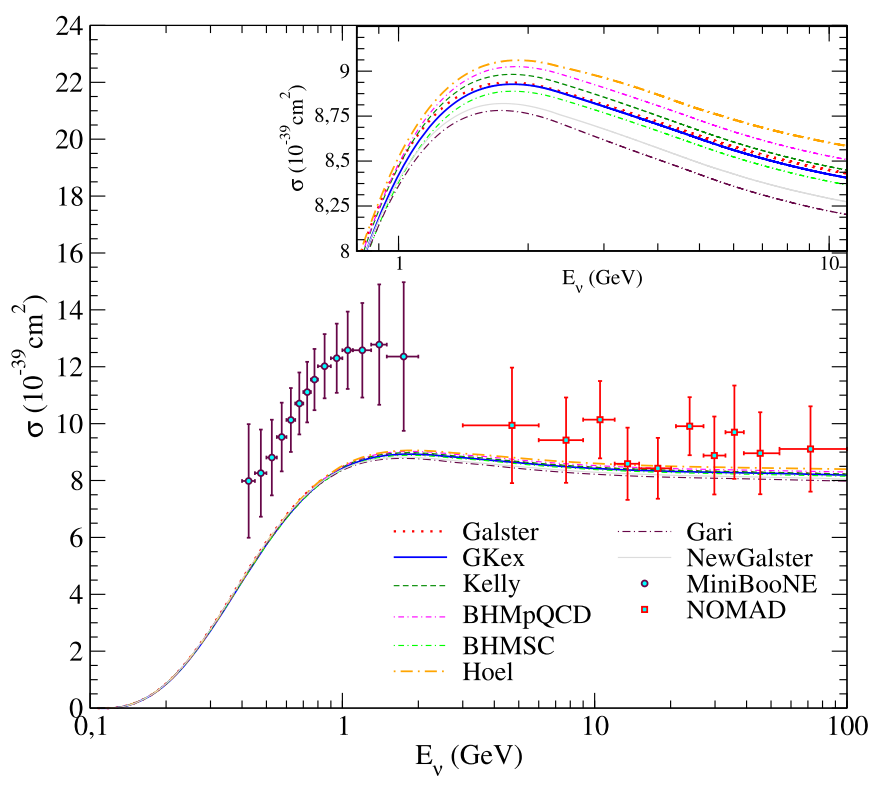

Fig. 4. (Color online.) CCQE $v_{\mu}{ }^{12} \mathrm{C}$ cross section per nucleon evaluated in the SuSA model for various parametrizations of the nucleon electromagnetic form factors.

this is where that term peaks, while the agreement with the antineutrino data would be less good.

We also show in Figs. 1 and 2 a line that represents the cross section computed with the SuSA model but where we have excluded all contributions coming from excitation energies below $50 \mathrm{MeV}$ to assess the importance of this region in the total cross section. As can be seen, this region is quite important even for very high neutrino energies (typically amounting to about $10 \%$ of the total). As noted above, the SuSA approach was not formulated to deal with such low-energy excitations and one might be concerned that the present modeling is spurious for these contributions. However, an alternative approach was taken long ago based on the excitation of discrete ph states in the regions up through where giant resonances dominate [30,31] and encouragingly the present SuSA model and those old results essentially agree, giving us confidence that the SuSA approach on the average does a rather good job even at such low excitation energies. It should also be mentioned that in [32] the contribution of the discrete excitations of the final nucleus ${ }^{12} \mathrm{~N}$ in $\mathrm{CC}$ neutrino scattering from ${ }^{12} \mathrm{C}$ was evaluated in a semi-relativistic shell model. The contribution from the discrete spectrum turned out to be below $2 \%$ for potential parameters fitted to reproduce the $Q$-value of the reaction.

In Figs. 1 and 2 the electromagnetic form factors of the nucleon entering in the vector charged current are those of the extended Gari-Krümpelmann (GKex) model of [33-35], whose validity extends over a wide range in the transferred momentum. In Fig. 4 we compare the SuSA results obtained with several other modern parametrizations of the form factors $G_{E}$ and $G_{M}$ [36]. In order to appreciate the dependence upon the vector form factors we do not show the error band in the SuSA result and instead have inserted a sub-panel zooming in on the region near the maximum. We observe that the uncertainties due to the electromagnetic nucleon form factors and the ones of the SuperScaling model are of the same order. Furthermore, all of the parametrizations are essentially equivalent for the kinematics that are relevant for these neutrino scattering experiments.

Next we explore the sensitivity of the cross sections to the axial-vector form factor. When employing a dipole parametrization for this the "standard" value of the axial-vector dipole mass is $M_{A}=1.032 \mathrm{GeV} / c^{2}$, whereas in analyzing the MiniBooNE data
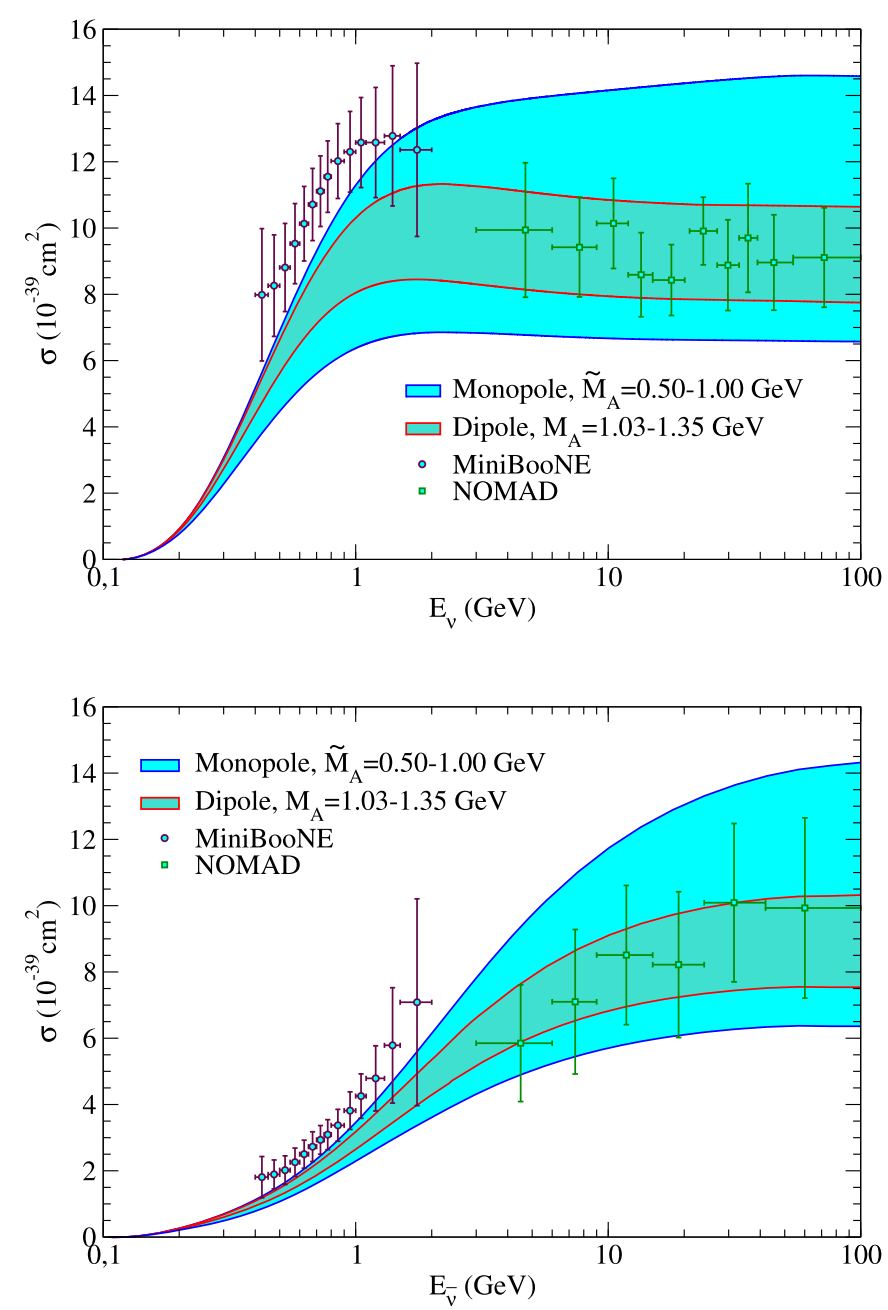

Fig. 5. (Color online.) CCQE $v_{\mu}-{ }^{12} \mathrm{C}$ (upper panel) and $\bar{v}_{\mu}-{ }^{12} \mathrm{C}$ (lower panel) cross section per nucleon evaluated in the SuSA model for monopole (blue outer band) and dipole (red inner band) parametrizations of the nucleon axial-vector form factor. A larger mass yields a higher cross section.

a large value of $M_{A}=1.35 \mathrm{GeV} / c^{2}$ was proposed [1]. The range spanned by these two is shown in Fig. 5 for neutrino and antineutrino scattering. Clearly the modified axial-vector mass produces an increase of the cross section that allows one to fit the lowenergy data in the RFG model, although the increase is too large to explain the data at high energy. Although phenomenologically successful, the dipole parametrization cannot be justified from a field-theoretical point of view [37] and it is well-known that, for instance in vector-meson dominance (VMD) models, the fact that at moderate momentum transfers the EM magnetic form factors are roughly dipole-like is a conspiracy involving the (monopole) $\rho$ and $\omega$ poles leading to an effective dipole behaviour (see, for example, the discussions in [35]). Therefore, in addition to the standard dipole form, we also consider a monopole form $G_{M}^{A}\left(Q^{2}\right)=$ $\left[1+Q^{2} / \tilde{M}_{A}^{2}\right]^{-1}$ motivated by VMD-based analyses such as those in $[33,34]$. Using the monopole axial-vector masses $\widetilde{M}_{A}=0.5 \mathrm{GeV} / c^{2}$ and $\widetilde{M}_{A}=1 \mathrm{GeV} / c^{2}$ (the range considered in [36]) employing the SuSA model we obtain the band also shown in Fig. 5. Note that increasing the axial-vector mass produces an increase of the cross sections with both parametrizations and a monopole axialvector form factor with $\widetilde{M}_{A} \simeq 1 \mathrm{GeV} / c^{2}$ leads to better agreement with both neutrino and antineutrino MiniBooNE cross sections. On the contrary, the same model overestimates significantly the higher-energy NOMAD data. In fact, the band width linked 


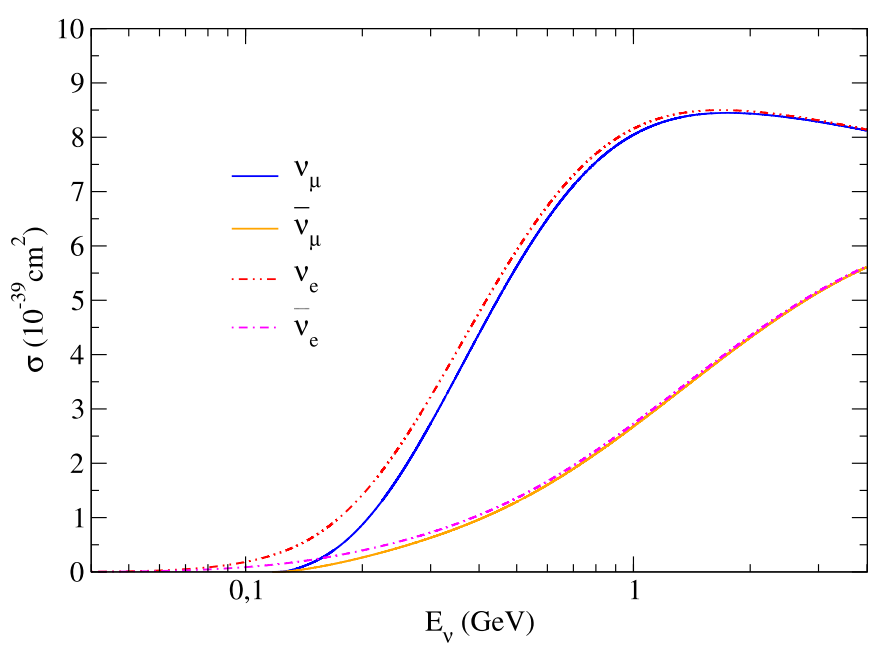

Fig. 6. (Color online.) SuSA predictions for muon (solid curves) and electron (dotted curves) neutrino and antineutrino CCQE cross section per nucleon on ${ }^{12} \mathrm{C}$.

to the two $\tilde{M}_{A}$-values used with the monopole axial-vector form factor is much larger than the one corresponding to the dipole parametrization. This is in accordance with previous results shown within the framework of parity-violating electron scattering [36]. We should notice that a dipole axial-vector form factor with $M_{A}=$ $1.35 \mathrm{GeV} / c^{2}$ (in the SuSA model) produces a cross section that is slightly lower in the MiniBooNE energy region than that obtained using $\widetilde{M}_{A}=1 \mathrm{GeV} / c^{2}$, but gives a "reasonable (or a better)" explanation of the NOMAD data. On the other hand, $\widetilde{M}_{A}=1 \mathrm{GeV} / c^{2}$ is probably not a good choice because the neutrino cross section keeps rising even at high energies. Indeed if one were to accept the monopole parametrization and fit the NOMAD data one would find that $\widetilde{M}_{A}=0.70 \pm 0.06(0.72 \pm 0.14) \mathrm{GeV} / c^{2}$ for neutrinos (antineutrinos). Old experiments with deuterium bubble chambers also performed fits of the data using a monopole axial form factor, obtaining $\widetilde{M}_{A}=0.57 \pm 0.05$ [38] and $\widetilde{M}_{A}=0.54 \pm 0.05$ [39]. While these studies would suggest that a dipole axial-vector form factor with the standard value of the dipole mass is preferred, given the modern interest in a potentially different behaviour, especially at high momentum transfers, new studies of neutrino disintegration of deuterium would be very valuable in clarifying this issue.

In Fig. 6 we compare the $v_{e}\left(\bar{v}_{e}\right)$ and $v_{\mu}\left(\bar{v}_{\mu}\right)$ cross sections in the SuSA model for the kinematics relevant for the proposed facility $\nu$ STORM [29], which will provide high quality electron neutrino beams in the energy range $E<4 \mathrm{GeV}$ for precise measurements of neutrino-nucleus cross sections. In particular, this could allow one to study the differences between muon and electron neutrino QE cross sections. Although the hadronic interaction is the same for $v_{\mu}$ and $v_{e}$, the different mass of the outgoing leptons produces a different energy transfer to the nucleus for the same incident neutrino energy. As seen in Fig. 6, this results in a small shift for low neutrino energy. For higher energies the small differences due to the lepton mass tend to disappear, yielding a universal curve, independent of the neutrino flavour. This is emphasized in Fig. 7, where the ratios between $v_{e}$ and $v_{\mu}$ (upper panel) and $\bar{v}_{e}$ and $\bar{v}_{\mu}$ (lower panel) cross sections in the SuSA and RFG models, as well as the double ratio SuSA/RFG, are shown. We note that the different models give the same results reaching unity for the ratio at energies above $1 \mathrm{GeV}$. For small energies one expects that the different nuclear excitation energy involved and the energy-dependence of the nuclear response functions will emphasize differences between the two cross sections, or between either of these and more sophisticated modeling of the low-lying nuclear excitations. A precise measurement of the cross sections in this region might therefore
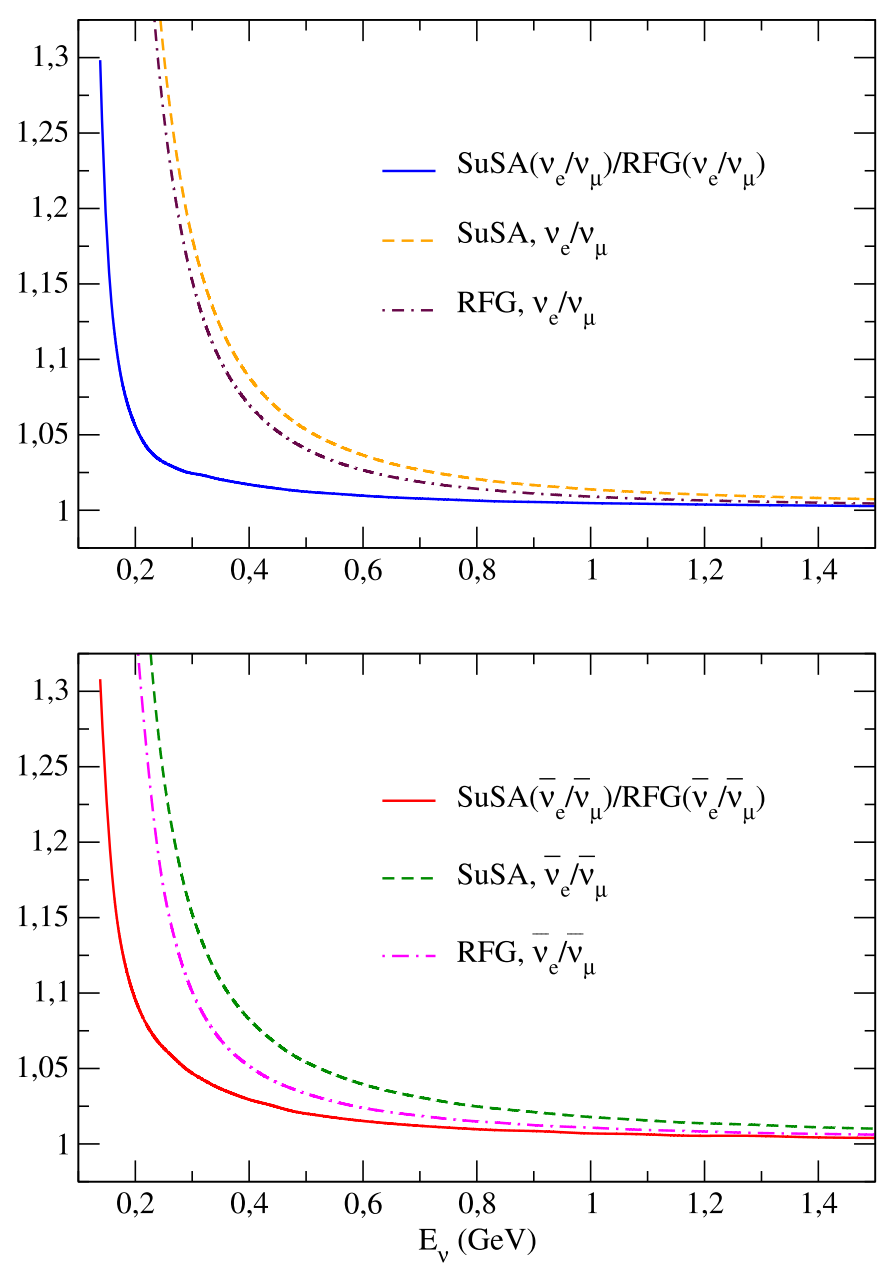

Fig. 7. (Color online.) Electron/muon neutrino (upper panel) and antineutrino (lower panel) $\mathrm{CCQE}$ cross section on ${ }^{12} \mathrm{C}$ evaluated in the SuSA and RGF models.

allow one to extract new information concerning the electroweak nuclear matrix elements. It should be noted that the differences between RFG and SuSA in $v_{e} / v_{\mu}\left(\bar{v}_{e} / \bar{v}_{\mu}\right)$ (Fig. 7) are caused, at least partially, by the different theoretical descriptions of the nuclear responses employed in these models, specifically that the RFG scaling function is bounded and does not extend to large and small values of the scaling variable.

Finally, we note that the difference between the two cases also to some degree arises not only via the different kinematics associated with the outgoing lepton mass, but also from Coulomb corrections, i.e., distortions of the final-state charged lepton wave functions in the Coulomb field of the nucleus. These are taken into account using the effective momentum approximation described in [28]. Their effects are found to be negligible in the energy range considered, becoming important only at neutrino energies below $200 \mathrm{MeV}$, where the cross sections are extremely small. Coulomb corrections have been incorporated in all results shown in Figs. 6 and 7.

\section{Conclusions}

In this Letter we have shown for the first time how the SuperScaling (SuSA) model behaves after being extended from intermediate to high energies as are relevant for recent neutrino scattering experiments. Comparisons are also made with the RFG model. Note that, although the differences between the RFG and SuSA predictions at high energy are small compared with the experimental 
error bars, the RFG fails to reproduce the $\left(e, e^{\prime}\right)$ data, whereas the SuSA model agrees by construction with the electron scattering $\mathrm{QE}$ cross section. Also note that, although the region of low excitation energy plays a significant role in the total cross section at all energies and consequently either of these models should be viewed with caution, since they are not well-suited to modeling the details of this region, in fact from comparisons with discrete-state modeling the SuSA approach does a reasonable job there when focusing on the total cross sections.

The SuSA model is expected to be robust enough to describe neutrino and antineutrino quasielastic cross sections in all of the experimentally available kinematic ranges, in the present study lacking only the two-body $2 \mathrm{p} 2 \mathrm{~h}$ MEC contributions that are expected to increase the cross sections by perhaps $10-15 \%$ or so. For the reasons stated above these are not included in the present work but will be added later once a robust approach to modeling them in the low- $q /$ low- $\omega$ region is in hand.

We have presented results for cross sections for MiniBooNE and NOMAD conditions, and have shown predictions corresponding to the $\nu$ STORM facility kinematics. The SuSA model has been used to investigate several aspects of the neutrino-nucleus interaction entering into the cross section, namely the impact from vector and axial-vector nucleon form factors and the dependence on the lepton flavour. The axial-vector form factor determines the strength of the axial-vector current matrix elements and crucially depends on the value of the axial-vector mass parameter. The dependence of the cross section upon this parameter is significant and yields uncertainties that are bigger than the other uncertainties of the model for high energies. Additionally, there is the issue of whether the axial-vector form factor should have a dipole or a monopole nature. Both types of behaviour have been explored in the present work.

\section{Acknowledgements}

This work was partially supported by DGI (Spain) grants FIS2011-28738-C02-01 and FIS2011-24149, by the Junta de Andalucia grants FQM-170 and FQM-225, by the Italian INFN under contract MB31, by the INFN-MICINN Collaboration agreement AICD-2011-0704, the Spanish Consolider-Ingenio 2000 programmed CPAN, and in part (T.W.D.) by US Department of Energy under cooperative agreement DE-FC02-94ER40818. G.D.M. acknowledges support from a fellowship from the Fundación Cámara (University of Sevilla). We thank R. González-Jiménez for providing the various parametrizations of the EM form factors and for useful discussions, and L. Alvarez-Ruso for critical reading of the manuscript and helpful suggestions.

\section{References}

[1] A.A. Aguilar-Arevalo, et al., MiniBooNE Collaboration, Phys. Rev. D 81 (2010) 092005, arXiv:1002.2680 [hep-ex].
[2] A.A. Aguilar-Arevalo, et al., MiniBooNE Collaboration, arXiv:1301.7067 [hepex].

[3] J.E. Amaro, M.B. Barbaro, J.A. Caballero, T.W. Donnelly, C.F. Williamson, Phys. Lett. B 696 (2011) 151, arXiv:1010.1708 [nucl-th].

[4] J.E. Amaro, M.B. Barbaro, J.A. Caballero, T.W. Donnelly, J.M. Udias, Phys. Rev. D 84 (2011) 033004, arXiv:1104.5446 [nucl-th].

[5] J.E. Amaro, M.B. Barbaro, J.A. Caballero, T.W. Donnelly, Phys. Rev. Lett. 108 (2012) 152501, arXiv:1112.2123 [nucl-th].

[6] O. Benhar, P. Coletti, D. Meloni, Phys. Rev. Lett. 105 (2010) 132301, arXiv: 1006.4783 [nucl-th].

[7] M. Martini, M. Ericson, G. Chanfray, J. Marteau, Phys. Rev. C 81 (2010) 045502 arXiv:1002.4538 [hep-ph].

[8] C. Juszczak, J.T. Sobczyk, J. Zmuda, Phys. Rev. C 82 (2010) 045502, arXiv: 1007.2195 [nucl-th].

[9] A.V. Butkevich, Phys. Rev. C 82 (2010) 055501, arXiv:1006.1595 [nucl-th].

[10] A. Meucci, C. Giusti, F.D. Pacati, Phys. Rev. D 84 (2011) 113003, arXiv:1110.3928 [nucl-th].

[11] A. Meucci, M.B. Barbaro, J.A. Caballero, C. Giusti, J.M. Udias, Phys. Rev. Lett. 107 (2011) 172501, arXiv:1107.5145 [nucl-th].

[12] A. Bodek, H.S. Budd, M.E. Christy, Eur. Phys. J. C 71 (2011) 1726, arXiv: 1106.0340 [hep-ph].

[13] J. Nieves, I. Ruiz Simo, M.J. Vicente Vacas, Phys. Lett. B 707 (2012) 72 arXiv:1106.5374 [hep-ph].

[14] O. Lalakulich, K. Gallmeister, U. Mosel, Phys. Rev. C 86 (2012) 014614, arXiv: 1203.2935 [nucl-th].

[15] J.T. Sobczyk, Phys. Rev. C 86 (2012) 015504, arXiv:1201.3673 [hep-ph].

[16] V. Lyubushkin, et al., NOMAD Collaboration, Eur. Phys. J. C 63 (2009) 355, arXiv: 0812.4543 [hep-ex].

[17] L. Fields, et al., MINERvA Collaboration, arXiv:1305.2234 [hep-ex].

[18] G.A. Fiorentini, et al., MINERvA Collaboration, arXiv:1305.2243 [hep-ex].

[19] D.B. Day, J.S. McCarthy, T.W. Donnelly, I. Sick, Ann. Rev. Nucl. Part. Sci. 40 (1990) 357.

[20] T.W. Donnelly, I. Sick, Phys. Rev. Lett. 82 (1999) 3212, arXiv:nucl-th/9809063.

[21] T.W. Donnelly, I. Sick, Phys. Rev. C 60 (1999) 065502, arXiv:nucl-th/9905060.

[22] C. Maieron, T.W. Donnelly, I. Sick, Phys. Rev. C 65 (2002) 025502, arXiv:nucl-th/ 0109032.

[23] C. Maieron, J.E. Amaro, M.B. Barbaro, J.A. Caballero, T.W. Donnelly, C.F. Williamson, Phys. Rev. C 80 (2009) 035504, arXiv:0907.1841 [nucl-th].

[24] T.W. Donnelly, J.W. Van Orden, T. De Forest Jr., W.C. Hermans, Phys. Lett. B 76 (1978) 393.

[25] J.W. Van Orden, T.W. Donnelly, Ann. Phys. 131 (1981) 451.

[26] A. De Pace, M. Nardi, W.M. Alberico, T.W. Donnelly, A. Molinari, Nucl. Phys A 726 (2003) 303, arXiv:nucl-th/0304084.

[27] J.E. Amaro, C. Maieron, M.B. Barbaro, J.A. Caballero, T.W. Donnelly, Phys. Rev. C 82 (2010) 044601, arXiv:1008.0753 [nucl-th].

[28] J.E. Amaro, M.B. Barbaro, J.A. Caballero, T.W. Donnelly, A. Molinari, I. Sick, Phys. Rev. C 71 (2005) 015501, arXiv:nucl-th/0409078.

[29] P. Kyberd, et al., vSTORM Collaboration, arXiv:1206.0294 [hep-ex].

[30] J.S. O'Connell, T.W. Donnelly, J.D. Walecka, Phys. Rev. C 6 (1972) 719.

[31] T.W. Donnelly, Prog. Part. Nucl. Phys. 13 (1985) 183.

[32] J.E. Amaro, M.B. Barbaro, J.A. Caballero, T.W. Donnelly, Phys. Rev. Lett. 98 (2007) 242501

[33] E.L. Lomon, Phys. Rev. C 64 (2001) 035204, arXiv:nucl-th/0104039.

[34] E.L. Lomon, Phys. Rev. C 66 (2002) 045501, arXiv:nucl-th/0203081.

[35] C. Crawford, et al., Phys. Rev. C 82 (2010) 045211.

[36] R. González-Jiménez, J.A. Caballero, T.W. Donnelly, Phys. Rep. 524 (2013) 1 arXiv:1111.6918 [nucl-th].

[37] P. Masjuan, E. Ruiz Arriola, W. Broniowski, Phys. Rev. D 87 (2013) 014005, arXiv:1210.0760 [hep-ph].

[38] N.J. Baker, A.M. Cnops, P.L. Connolly, S.A. Kahn, H.G. Kirk, M.J. Murtagh, R.B. Palmer, N.P. Samios, et al., Phys. Rev. D 23 (1981) 2499.

[39] K.L. Miller, S.J. Barish, A. Engler, R.W. Kraemer, B.J. Stacey, M. Derrick, E. Fernandez, L. Hyman, et al., Phys. Rev. D 26 (1982) 537. 